\title{
Improved sensitivity using liquid chromatography mass spectrometry (LC-MS) for detection of propyl chloroformate derivatised $\beta-N$-methylamino-L-alanine (BMAA) in cyanobacteria
}

\author{
M Esterhuizen-Londt, S Downing and TG Downing* \\ Department of Biochemistry and Microbiology, Nelson Mandela Metropolitan University, PO Box 77000, \\ Port Elizabeth 6031, South Africa
}

\begin{abstract}
$\beta$ - $N$-methylamino-L-alanine (BMAA) is a difficult molecule to detect, primarily due to its presence in low concentrations in complex matrices. This has resulted in contradictory reports on the presence of BMAA in cyanobacteria. We report improved sensitivity of detection using propyl chloroformate derivatisation, liquid chromatographic (LC) separation, and single quadrupole mass spectrometry (MS) detection. Triple quadrupole mass spectrometry (MS/MS) was used to confirm the identity of BMAA in cyanobacteria based on product ions. We show a 10 -fold increase in sensitivity with the LC-MS method compared to the previously published gas chromatography mass spectrometry (GC-MS) method with pre-column derivatised BMAA using a commercially available amino acid derivatisation kit. Clear chromatographic separation of BMAA from 2,4-diaminobutyric acid (DAB), as well as the 20 standard amino acids, was achieved. The analytical method was validated by multiple derivatisation of samples, multiple users, and multiple injections, as well as in various matrices. The quantifier ion used was [M+ H] $]^{+}=333 \mathrm{~m} / z$. The MS/MS product ions $273 \mathrm{~m} / \mathrm{z}$ and $245 \mathrm{~m} / \mathrm{z}$ were used in identification and peak confirmation. Additionally, we confirm the presence of BMAA in cyanobacteria previously screened with GC-MS as well as the presence of BMAA in newly isolated cultures.
\end{abstract}

Keywords: $\beta$ - $N$-methylamino-L-alanine, BMAA, LC-MS, LC-MS/MS

\section{Introduction}

The non-proteinogenic amino acid $\beta$ - $N$-methylamino-L-alanine (BMAA, Fig. 1) is an excitatory neurotoxin, causing neurodegeneration as a result of selective motor neuron death, at concentrations as low as $30 \mu \mathrm{M}$ (Rao et al., 2006), and damage to motor neurons at concentrations of $10 \mu \mathrm{M}$ in the presence of other neurotoxic compounds (Lobner et al., 2007). BMAA was first identified in the seeds of Cycas micronesica, an indigenous cycad on the island of Guam (Vega and Bell, 1967), where it was initially implicated in the high incidence of amyotrophic lateral sclerosis-Parkinsonism dementia complex (ALS/PDC) (Cox et al., 2005). The link between ALS/PDC and BMAA was based on the presence of free and protein-associated BMAA in the brain tissue of 6 ALS/PDC patients from Guam, but in only 1 of 2 asymptomatic Chamorro brains and not in brain tissues from fatalities of unrelated diseases (Murch et al., 2004a). In support of these data, Pablo et al. (2009) detected BMAA in brain tissue samples of sporadic Alzheimer's disease and ALS but not in Huntington's disease post-mortem samples.

BMAA produced by symbiotic cyanobacteria (Nostoc) in Cycas micronesica coralloid roots (Cox et al., 2003) was consumed by Chamorro people in the form of flour products made from the cycad seeds. The flour was not considered an adequate source of BMAA as it was washed to remove free cycasin (a cycad glycoside toxin), which also removed free BMAA

\footnotetext{
To whom all correspondence should be addressed.

+2741 504-2359; fax: +2786 614-7129;
}

要

e-mail: tdowning@nmmu.ac.za

Received 19 July 2010; accepted in revised form 1 February 2011.<smiles>CNCC(N)C(=O)O</smiles>

BMAA<smiles>NCC[C@H](N)C(=O)O</smiles>

2,4-DAB
Figure 1

Chemical structures of BMAA and 2,4-diaminobutyric acid (DAB)

(Duncan et al., 1990). However, protein-associated BMAA was not taken into account (Murch et al., 2004b). Additionally, flying foxes (Pteropus mariannus), which are also consumed by Chamorro's, forage on the seeds of the cycads resulting in biomagnification of BMAA and exposure of consumers to elevated BMAA concentrations (Cox and Sacks, 2002; Banack et al., 2006). Montine et al. (2005), using an alternative analytical method, reported that no BMAA was detected in the brain tissue of Chamorro ALS/PDC patients. These non-replicated data from a single investigation cast doubt on the BMAA-ALS/ PDC link. However, further evidence supporting the link between BMAA and ALS/PDC came in the form of the sudden decline in ALS/PDC occurrence on Guam with a decline in the consumption of flying fox, due to the animal's near extinction (Monson et al., 2003). BMAA was subsequently found in the brain tissue of Canadian Alzheimer's patients (Murch et al., 2004a), suggesting alternate sources of BMAA in the food chain. However, Snyder et al. (2010) reported the lack of BMAA in human brain samples using a 2-dimensional gas chromatography coupled with time-of-flight mass-spectrometry analysis followed by a targeted Parallel Factor Analysis 
deconvolution method. Similarly, several groups have been unable to detect BMAA in complex matrices (Rosén and Hellenäs, 2008; Kushnir and Bergquist, 2009).

Cox et al. (2005) found that culture collection strains of free-living freshwater and marine cyanobacteria from all five taxonomic sections contained BMAA, and concluded that most, if not all, cyanobacteria produce BMAA. Findings by Cox et al. (2005) were confirmed by Banack et al. (2007), Esterhuizen and Downing (2008), Metcalf et al. (2008) and Faassen et al. (2009). Metcalf et al. (2008) and Faassen et al. (2009) reported the presence of BMAA in cyanobacterial surface scum therefore accentuating the risk of human exposure to BMAA. However, the extent of exposure is currently unknown. Recent publications suggest that BMAA is not only present in cyanobacteria, but may also be released into water supplies and reservoirs used by humans (Metcalf et al., 2008; Banack et al., 2007).

There has been considerable controversy over the analytical methods employed for BMAA detection and its role in ALS/ PDC. Additionally, the controversy over analysis has resulted in some debate over the validity of the reports on cyanobacteria as the source of BMAA. To date several analytical methods have been published that employ pre-column derivatisation with either ethyl chloroformate or 6-aminoquinolyl- $N$-hydroxysuccinimidyl carbamate (AQC) and analysis by UPLC-UV, HPLC-FLD, LC-MS, LC-MS/MS and GC-MS (Banack et al., 2007; Cox et al., 2003, 2005; Esterhuizen and Downing 2008; Spáčil et al., 2010). Post-column derivatisation with ninhidrin and analysis by amino acid analyser (Banack et al., 2007), and methods employing no derivatisation with HILIC LC-MS/MS, have also been reported (Rosén and Hellenäs, 2008; Kubo et al., 2007; Moura et al., 2009; Krüger et al., 2010; Faassen et al., 2009). Only one account where pre-column derivitisation was not used prior to MS detection reported the presence of BMAA in cyanobacteria, but at much lower concentrations than reported with pre-column derivatisation (Faassen et al., 2009).

Recently, Rosén and Hellenäs (2008) reported the cooccurrence of BMAA and DAB in cycad seed and the sole occurrence of DAB in cyanobacterial samples. DAB is a structural analogue of BMAA. Like BMAA, DAB is a neurotoxin, and was first isolated from Lathyrus latifolius. Rosén and Hellenäs (2008) suggested that previous reports of BMAA in cyanobacteria might be due to confusion with DAB. Similarly, Krüger et al. (2010) suggested misidentification of DAB and/ or tryptophan as BMAA in cyanobacteria. However, Faassen et al. (2009) and Spáčil et al. (2010) reported co-occurrence of BMAA and DAB in various cyanobacterial samples tested.

In order to improve sensitivity, and in effect improve separation of confounder molecules, LC-MS separation with propyl chloroformate pre-column derivatisation was employed to confirm previous reports, screen additional samples, and show adequate distinction between DAB, BMAA and tryptophan in these samples.

\section{Experimental}

Chemicals were purchased from Sigma-Aldrich. Stock solutions of BMAA (Sigma-Aldrich, L-BMAA hydrochloride B-107) and DAB (Fluka, L-2-4-diaminobutyric acid hydrochloride \#32830) were prepared to a concentration of $100 \mathrm{mM}$ by mixing individual standards in Millipore $(16.8 \mathrm{M} \Omega)$ water. The EZ:Faast ${ }^{\mathrm{TM}}$ LC-MS Free (Physiological) Amino Acids Kit with $250 \times 2.0 \mathrm{~mm}$ column (Phenomenex KH0-7337) was purchased from Separations, South Africa.

Cyanobacterial cultures representing taxonomic diversity and geographic distribution in Southern Africa were collected from various freshwater impoundments from 2006 to 2007 and made uni-algal by standard methods on BG-11 and BG-11, solid media (Rippka, 1988). Cultures were maintained in liquid media at a temperature of $23^{\circ} \mathrm{C}\left( \pm 1^{\circ} \mathrm{C}\right)$ at a light intensity of 30 to $40 \mu \mathrm{mol}$ photon $\mathrm{m}^{2} \mathrm{~s}^{-1}$ under constant illumination (Triton Dayglo(C). Culture purity was confirmed microscopically before BMAA analysis.

The extraction method was adapted from Cox et al. (2003). Cyanobacterial cultures were centrifuged using a Beckmann Avanti J-20 centrifuge at $15800 \mathrm{G}$ at $4^{\circ} \mathrm{C}$ for $10 \mathrm{~min}$ to collect biomass, which was snap-frozen in liquid nitrogen and lyophilised overnight in a VirTis bench-top freeze dryer (condenser temperature of $-51^{\circ} \mathrm{C}$ and a vacuum of 350 mTorr). BMAA was extracted from lyophilised cultures (20-500 mg dry weight) by sonication (Bandelin sonoplus ultrasonic sonicator, $40 \%$ power at $1 \times 30 \mathrm{~s}$ burst, and a $50 \%$ duty cycle pulse) with $0.1 \mathrm{M}$ trichloroacetic acid. Free BMAA was obtained in the supernatant after centrifugation (Beckmann Avanti J-20 at $15800 \mathrm{G}$ for $3 \mathrm{~min}$ at $\left.4^{\circ} \mathrm{C}\right)$ to precipitate proteins. TCA $(0.1 \mathrm{M})$ was added to the pellet again to wash off residual free BMAA. The pellet was re-suspended by repeated vortexing and centrifuged as before. Supernatants were pooled and analysed for free BMAA. Proteinassociated BMAA was released by liquid acid hydrolysis in $6 \mathrm{M}$ $\mathrm{HCl}$ and $2 \%$ thioglycolic acid (to prevent tryptophan oxidation) at $110^{\circ} \mathrm{C}$ for $24 \mathrm{~h}$ in an inert atmosphere and filtered through a $0.22 \mu \mathrm{m}$ filter (Lasec) to remove debris prior to derivatisation.

The EZ:Faast ${ }^{\mathrm{TM}}$ amino acid analysis for LC-MS derivatisation incorporates a concentration step on a proprietary sorbent medium to eliminate the effects of interfering compounds, a wash step, elution from, and removal of, the sorbent medium, and sample clean-up by organic extraction as well as derivatisation with a proprietary chloroformate derivative. Figure 2 shows the simplified schematic illustration of how the derivatisation reaction occurs and the BMAA derivative obtained using propyl chloroformate.

BMAA, DAB, tryptophan, and proteinogenic amino acids were analysed using a Shimadzu LC coupled to a Shimadzu MS (2010 EV) after derivatisation using the LC form of the EZ:Faast ${ }^{\mathrm{TM}}$ amino acids analysis kit (Phenomenex). BMAA was separated from other amino acids by liquid chromatography on a commercial column (Phenomenex AAA-MS $250 \times 2.0 \mathrm{~mm}$<smiles>CCCOC(=O)NC(CN(C)C(=O)OCCC)C(=O)O[14C](=O)[O-]</smiles>

Figure 2

Schematic diagram of the BMAA derivatisation reaction 
amino acid analysis column). A solvent gradient was used with A: $10 \mathrm{mM}$ ammonium formate in water and B: $10 \mathrm{mM}$ ammonium formate in methanol $(0.0 \mathrm{~min}=68 \% \mathrm{~B}, 13.00 \mathrm{~min}=83 \%$ $\mathrm{B}, 13.01 \mathrm{~min}=68 \% \mathrm{~B}, 17.00 \mathrm{~min}=68 \% \mathrm{~B}$ ) at a flow rate of 0.25 $\mathrm{m} \ell \cdot \mathrm{min}^{-1}$ and $1 \mu \ell$ sample injection volume. Column temperature was kept constant at $35^{\circ} \mathrm{C}$. The mass spectrometer electrospray ionisation (ESI) source (positive ion mode) temperature was set at $250^{\circ} \mathrm{C}$. The ion scan range was between 20 and $600 \mathrm{~m} / \mathrm{z}$. The detector voltage was set at $1.5 \mathrm{kV}$. The interface voltage was set at $4.5 \mathrm{kV}$ and the curved desolvation line voltage at $-20 \mathrm{~V}$ with the heating block at $200^{\circ} \mathrm{C}$. The data was analysed using LC-MS solutions Ver. 3 software.

LC-MS/MS was used to distinguish between DAB, BMAA and tryptophan, which all yield quasi-molecular ions at $\mathrm{m} / \mathrm{z} 333$ upon propyl chloroformate derivatisation. For LC-MS/MS, samples of free and protein-associated BMAA extracts were derivatised using propyl chloroformate as previously described and separated by HPLC (Water Acquity Ultra Performance LC) on a Phenomenex EZ:Faast AAA-MS column $(250 \times 2.0 \mathrm{~mm})$ by gradient elution $(0.00 \mathrm{~min}=68 \% \mathrm{~B}, 13.00 \mathrm{~min}=83 \% \mathrm{~B}, 13.01 \mathrm{~min}$ $=68 \% \mathrm{~B}, 17.00 \mathrm{~min}=68 \% \mathrm{~B}$ ) with a mobile phase composition of $10 \mathrm{mM}$ ammonium formate in water (A) and $10 \mathrm{mM}$ ammonium formate in methanol (B) (flow rate: $0.25 \mathrm{~m} \ell \cdot \mathrm{min}^{-1}$; column temperature: $35^{\circ} \mathrm{C}$ ). Derivatised samples of free and proteinassociated cellular amino acids were analysed using a Thermo Finnigan TSQ Quantum triple quadrupole mass spectrometer with the following settings: ESI voltage set to 5000 , nebulising gas (Nitrogen) at a flow rate of 40, vaporisation temperature of $199^{\circ} \mathrm{C}$, capillary temperature at $270^{\circ} \mathrm{C}$ and capillary and tube lens offsets set to 35 and 70, respectively. Collision-induced dissociation was achieved within the second quadrupole with the precursor ionspecific collision energy set at 12 for the $333 \mathrm{~m} / \mathrm{z}$ precursor ion, and argon gas supplied at 1 mTorr.

The single quadrupole detector (Shimadzu 2010EV) with a Shimadzu LC-20AB liquid chromatography system was validated using a propyl chloroformate pre-column derivatisised BMAA standard (Sigma). The resulting molecule was quantified against 3 internal standards: homoarginine (HARG), methionine-D3 (Met-D3) and homophenylalanine (HPHE). Multiple user, delayed derivatisation and delayed analysis assessments were conducted to verify the system and to determine how robust the method was. BMAA standards were derivatised in varying concentrations on one day and injected in triplicate to assess equipment accuracy and reproducability, and derivatisation of standards in triplicate on 3 consecutive days and by 3 individuals was undertaken in order to determine derivatisation reproducibility. E. coli and BSA were used as negative controls for free and protein-associated BMAA, respectively. A matrix of 20 standard amino acids was spiked with the BMAA authenticated standard to test for adequate separation. A calibration curve was constructed for quantification based on the ratio of peak areas of the representative molecular ion for BMAA $(\mathrm{m} / \mathrm{z}=333)$ to that of Met-D3 $(\mathrm{m} / \mathrm{z}=$ 281). All analyses were conducted in triplicate. Lower limit of detection (LLOD) and lower limit of quantification (LLOQ) values were determined experimentally by dilution to invisibility $(\mathrm{S} / \mathrm{N}<2$ in a matrix) and reproducibility $(\mathrm{SD} \pm 5 \%$ in a matrix) respectively.

Accuracy and precision was calculated from the 5 calibration standards of BMAA against the internal standard to validate the method (Table 1). Precision represents a percentage relative standard deviation (\%RSD) of the analyte to internal standard peak areas obtained from replicates $(n=5)$. Accuracy was calculated as a percentage variation relative to the nominal concentration of each point.

\begin{tabular}{|c|c|c|c|c|}
\hline \multicolumn{5}{|c|}{ Table 1 } \\
Precision and accuracy of calibration standards \\
\hline $\begin{array}{c}\text { Calibration } \\
\text { level }\end{array}$ & $\begin{array}{c}\text { BMAA } \\
\text { (mg/ })\end{array}$ & SD & \%RSD & $\begin{array}{c}\text { Accuracy } \\
(\%)\end{array}$ \\
\hline 1 & 0.1489 & 0.0013 & 4.7815 & 87.9 \\
\hline 2 & 0.2977 & 0.0002 & 0.6015 & 94.6 \\
\hline 3 & 0.5955 & 0.0038 & 3.6981 & 98.9 \\
\hline 4 & 1.191 & 0.0029 & 1.7383 & 92.7 \\
\hline 5 & 2.382 & 0.0045 & 1.4093 & 97.9 \\
\hline
\end{tabular}

A
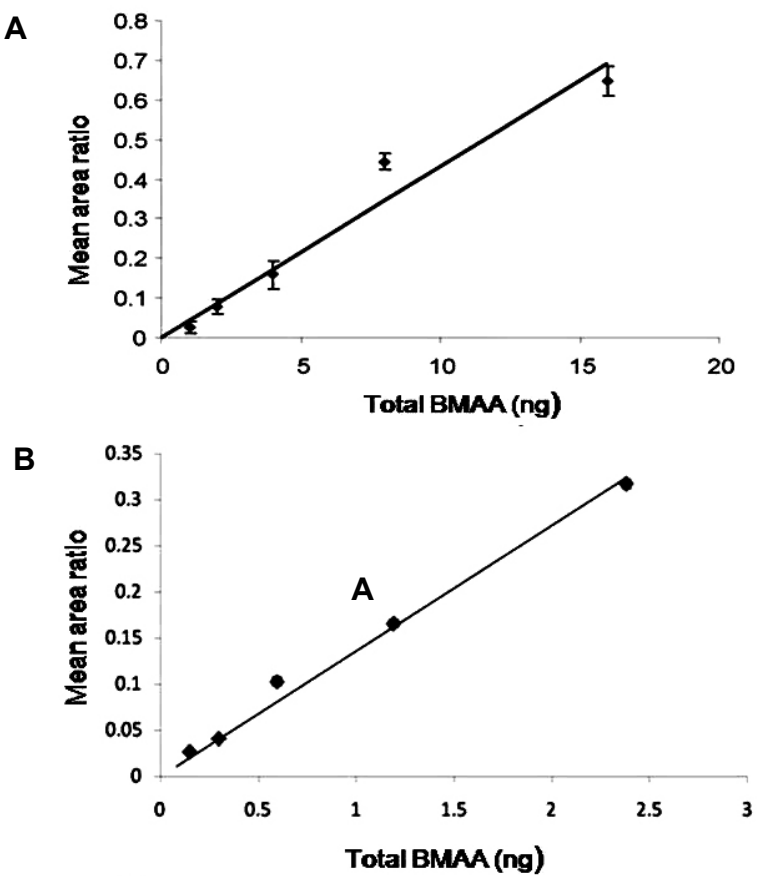

C

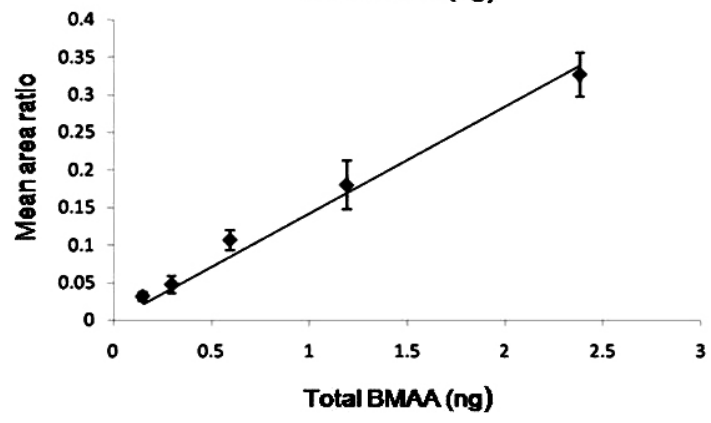

Figure 3

Calibration curve of BMAA standard against the appropriate internal standard. $(A)$ is the standard curve obtained off a Thermofinnigan GC-MS against internal standard of norvaline (NORV), (B) shows the standard curve evaluating the Shimadzu LC-MS machine reproducibility against ISTD methionine-D3 $\left(n=5, r^{2}=0.989\right)$ and $(C)$ shows user validation for derivatisation and analysis on Shimadzu LC-MS $\left(n=5, r^{2}=0.985\right)$.

\section{Results and discussion}

Analytical reproducibility was excellent with insignificant variation in individual quantification runs on a given sample $\left(r^{2}=0.989, n=5\right)$. Derivatisation reproducibility across the concentration range used for the calibration curve was acceptable $\left(r^{2}=0.985, n=5\right)$. Figure 3 shows the standard curves obtained using commercial BMAA standard on Thermofinnigan GC-MS (A) and Shimadzu LC-MS for 
analysis of intra-day (B) and inter-day (C) variation.

The lower limits of sensitivity of detection and quantification of an authenticated standard for GC-MS analysis were $0.0042 \mathrm{nmol}$ per injection and $0.084 \mathrm{nmol}$ per injection, respectively (Esterhuizen and Downing, 2008). The lower limit of detection in a matrix of $20 \mu \mathrm{M}$ for each of the 20 standard amino acids was $0.0252 \mathrm{nmol}$ per injection. For the LC-MS method reported here, an LLOD of 0.042 pmol per injection
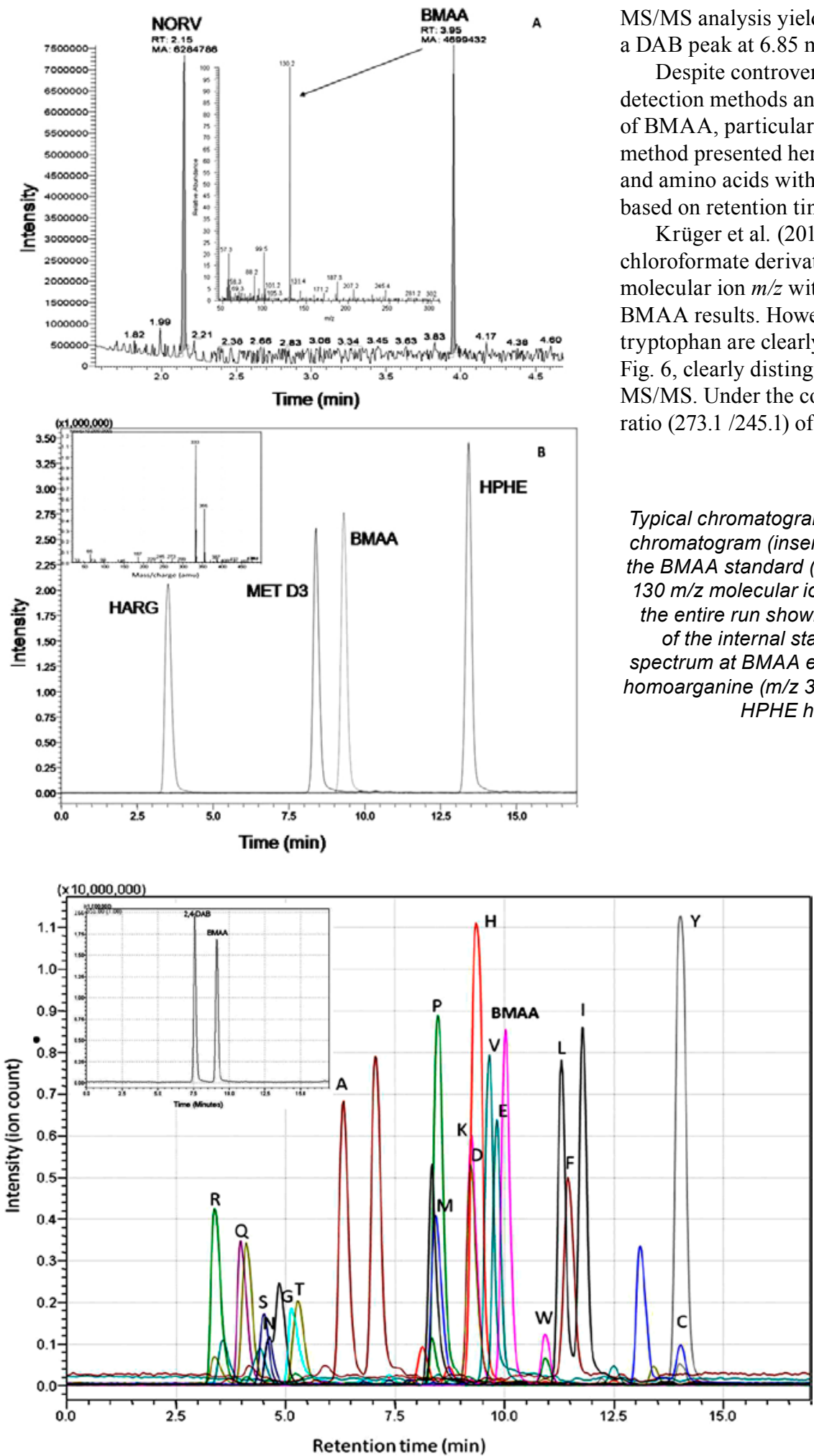

and LLOQ of 0.82 pmol per injection (S/N 2.07) was achieved. The LC-MS method improved the sensitivity 10-fold. LC-MS/ MS with single reaction monitoring (SRM) for product ions $273,245 \mathrm{~m} / \mathrm{z}$ further increased the sensitivity.

Figure 4 shows typical chromatograms obtained for each analytical method. With analysis by GC-MS (A), BMAA had a retention time of $3.95 \mathrm{~min}$ and a $\mathrm{m} / \mathrm{z}$ of 130.2. LC-MS analysis (B) yielded a peak at retention time of 9.4 min with a quasimolecular ion $\mathrm{m} / \mathrm{z}$ of 333 on the Shimadzu LC-MS. Minor variation in retention time in complex matrices was observed. MS/MS analysis yielded a BMAA peak with a RT of $7.37 \mathrm{~min}$, a DAB peak at $6.85 \mathrm{~min}$ and tryptophan at $7.95 \mathrm{~min}$.

Despite controversy regarding the validity of BMAA detection methods and possible misidentification of isomers of BMAA, particularly DAB (Krüger et al., 2010) (Fig. 1), the method presented here clearly distinguished between isomers and amino acids with the same quasi-molecular ion (Fig. 5), based on retention time using LC-MS.

Krüger et al. (2010) also suggest that tryptophan, the propyl chloroformate derivative of which shares the same quasimolecular ion $m / z$ with BMAA, could lead to false positive BMAA results. However, as seen in Fig. 5, DAB, BMAA and tryptophan are clearly distinguishable by the RT and, as seen in Fig. 6, clearly distinguishable by the ratio of product ions using MS/MS. Under the conditions used, DAB has a product ion $\mathrm{m} / \mathrm{z}$ ratio $(273.1 / 245.1)$ of greater than 2; for BMAA the ratio was

\section{Figure 4 (left)}

Typical chromatograms of isolates. (A) Thermofinnigan GC-MS chromatogram (insert is mass spectrum at the retention time of the BMAA standard (Esterhuizen and Downing, 2008). Note the $130 \mathrm{~m} / \mathrm{z}$ molecular ion. (B) Shimadzu LC-MS chromatogram of the entire run showing only the selected $\mathrm{m} / \mathrm{z}$ chromatograms of the internal standards and BMAA (insert shows mass spectrum at BMAA elution time). Internal standards are HARG, homoarganine ( $\mathrm{m} / \mathrm{z}$ 317); Met-D3, methionine-D3 ( $\mathrm{m} / \mathrm{z} 281)$; and HPHE homo-phenylalanine $(\mathrm{m} / \mathrm{z}$ 308).

Figure 5 (left)

SPE recovery of BMAA from an amino acid matrix, detected using a Shimadzu 2010EV LC-MS single quadrupole for propylchloroformate derivatised amino acids. Detection by $M S$ was as follows: Arginine (R) $303 \mathrm{~m} / \mathrm{z}$, Glutamine (Q) 275 $\mathrm{m} / \mathrm{z}$, Serine (S) $234 \mathrm{~m} / \mathrm{z}$, Glycine (G) 204 $\mathrm{m} / \mathrm{z}$, Threonine $(T) 248 \mathrm{~m} / \mathrm{z}$, Alanine $(A)$ $218 \mathrm{~m} / \mathrm{z}$, Proline $(P) 244 \mathrm{~m} / \mathrm{z}$, Methionine (M) $278 \mathrm{~m} / \mathrm{z}$, Lysine (K) $361 \mathrm{~m} / \mathrm{z}$, Aspartate (D) $304 \mathrm{~m} / \mathrm{z}$, Histidine (H) 370, Valine (V) $246 \mathrm{~m} / \mathrm{z}, \beta-\mathrm{N}$ methylamino-Lalanine (BMAA) $333 \mathrm{~m} / \mathrm{z}$, Glutamic acid (E) $318 \mathrm{~m} / \mathrm{z}$, Tryptophan (W) $333 \mathrm{~m} / \mathrm{z}$, Leucine (L) $260 \mathrm{~m} / \mathrm{z}$, Phenylalanine ( $F$ ) $294 \mathrm{~m} / \mathrm{z}$, Isoleucine (I) $260 \mathrm{~m} / \mathrm{z}$, Tyrosine (Y) $396 \mathrm{~m} / \mathrm{z}$. Cysteine (C) $336 \mathrm{~m} / \mathrm{z}$. INSERT: Shimadzu 2010EV MS single quadrupole detection of $L C$ separated propyl chloroformate derivatised BMAA

(RT $9.1 \mathrm{~min}$ ) and DAB (RT $7.6 \mathrm{~min})$ using a $250 \times 2.0 \mathrm{~mm}$ EZ:faast ${ }^{T M} A A A$ LC column (Phenomenex). Minor ions used for confirmation. $\mathrm{m} / \mathrm{z} 245$ used as qualifier ion for $B M A A$ as it is unique to $B M A A(\mathrm{~m} / \mathrm{z} 333)$ and ratio of 273 used for confirmation of $D A B(\mathrm{~m} / \mathrm{z} 333)$. 


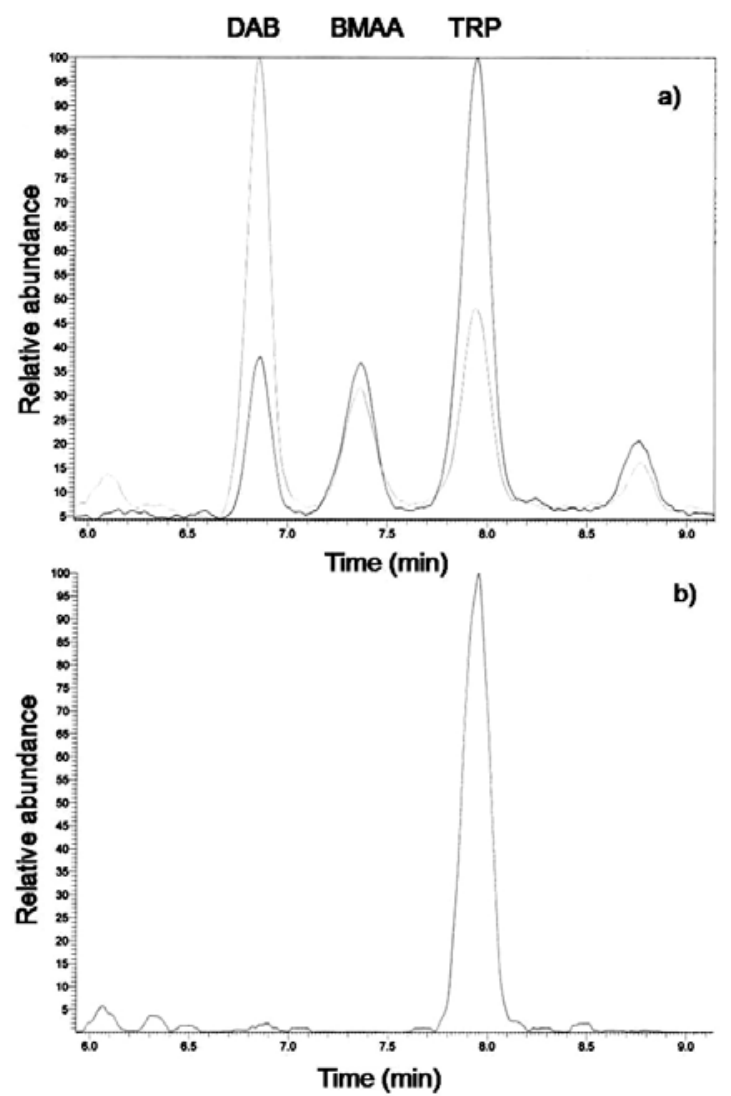

Figure 6

MS/MS chromatograms of product ions (a) $\mathrm{m} / \mathrm{z} 273$ (light grey) and $\mathrm{m} / \mathrm{z} 245$ (dark grey) , and $b$ ) $\mathrm{m} / \mathrm{z} 230$, of the precursor ion $\mathrm{m} / \mathrm{z}$ 333. DAB, BMAA and tryptophan can be clearly distinguished by product ion ratios (DAB has $273>245$, BMAA has $273 \approx 245$ and tryptophan has $273<245$ and a unique product ion of $\mathrm{m} / \mathrm{z} 230$.

greater than 0.7 and less than 1.2 , whereas for tryptophan the ratio was always less than 0.6 .

The derivatisation and detection method described here is available as a standardised kit that is both simple and rapid as well as being extremely sensitive. Final sensitivity is equipment-dependent but detection of BMAA at concentrations below $100 \mathrm{ng} \ell^{-1}$ should be achievable based on an on-column LLOQ of 0.84 pmol per injection and the solid phase derivatisation, elution and re-suspension system that can concentrate up to 150 times. Additionally, the derivatised samples are stable for several hours. The initial adsorption step takes place on a small quantity of the material, however, possibly limiting use to small volumes or previously concentrated samples. However, the SPE sorbent tip results in additional sample clean-up, together with a wash step and an organic sample extraction. The advantage of the additional purification step is high column efficiency and resolution. This provides complete baseline separation for easy quantitation. Internal standards are used for improved quantitation. The kit facilitates high reproducibility within and between laboratories. Multi-user verification of the method (Fig. 3) yielded a high level of reproducibility.

\section{South African cultures}

The presence of BMAA in cyanobacteria, as reported by Esterhuizen and Downing (2008), was confirmed using the

\begin{tabular}{|c|c|c|c|c|}
\hline \multicolumn{5}{|c|}{$\begin{array}{c}\text { Table } 2 \\
\text { The BMAA content of free and protein-associated BMAA } \\
\text { in cultures of free-living freshwater South African } \\
\text { cyanobacterial isolates not previously tested }\end{array}$} \\
\hline $\begin{array}{l}\text { Cyanobacterial } \\
\text { strain/species }\end{array}$ & $\begin{array}{c}\text { Section } \\
\text { (Rippka, } \\
\text { 1988) }\end{array}$ & $\begin{array}{c}\text { Origin/ } \\
\text { Catchment }\end{array}$ & $\begin{array}{c}\text { Free } \\
\text { BMAA } \\
(\mu \mathrm{g} / \mathrm{g}) \\
\end{array}$ & $\begin{array}{l}\text { Bound } \\
\text { BMAA } \\
(\mu \mathrm{g} / \mathrm{g}) \\
\end{array}$ \\
\hline Microcystis & $\mathrm{I}$ & $\mathrm{C} 811$ & 0.135 & NQ \\
\hline Synechocystis & $\mathrm{I}$ & PCC6803 & ND & NQ \\
\hline Synechocystis & $\mathrm{I}$ & C522 & 0.126 & ND \\
\hline Synechocystis & $\mathrm{I}$ & M111 & 0.391 & 1.290 \\
\hline Oscillatoria & III & $\mathrm{C} 421$ & NQ & 1.227 \\
\hline Oscillatoria & III & C521 & 0.976 & 0.936 \\
\hline Oscillatoria & III & C523 & ND & 1.48 \\
\hline Oscillatoria & III & D231 & NQ & 0.0518 \\
\hline Oscillatoria & III & D237 & 0.058 & 0.179 \\
\hline Oscillatoria & III & D241 & 0.116 & 10.616 \\
\hline Oscillatoria & III & G405 & ND & 0.751 \\
\hline Oscillatoria & III & $\mathrm{J} 121$ & 0.201 & ND \\
\hline Pseudoanabaena & III & A213 & NQ & ND \\
\hline Pseudoanabaena & III & C321 & 0.876 & NQ \\
\hline Calothrix & IV & D242 & 0.050 & ND \\
\hline Calothrix & IV & C522 & 0.060 & NQ \\
\hline Anabaena & IV & A224 & 0.156 & ND \\
\hline Anabaena & IV & A311 & NQ & ND \\
\hline Anabaena & IV & V202 & $\mathrm{ND}$ & ND \\
\hline Anabaena & IV & V203 & 0.577 & 1.393 \\
\hline
\end{tabular}

propyl chloroformate derivatisation and single quadrupole MS detection after LC separation. Table 2 shows the BMAA content for a range of South African isolates from the cyanobacterial culture collection which had not been previously tested, representing both taxonomic and geographical diversity.

Previously, BMAA was detected in $96 \%$ ( $\pm 2 \%$ ) of all strains examined (Cox et al., 2005; Esterhuizen and Downing, 2008). With LC-MS detection, BMAA was only detected in $80 \%(16 / 20)$ of freshwater isolates. This difference is, however, statistically insignificant given the limited number of strains tested. Preliminary investigations into the modulation of the BMAA content of cyanobacteria suggest highly variable BMAA concentration as a function of environmental conditions and/or culture age and history (data not shown). This may also explain the reduced percentage of strains with detectable BMAA

The presence of BMAA in a primary producer in aquatic ecosystems raises the question of possible bioaccumulation and biomagnification, as occurs in the Guam ecosystem (Banack et al., 2006, 2007; Banack and Cox, 2003). This possibility increases the risk of human and animal exposure. Rao et al. (2006) and Lobner et al. (2007) showed that very low concentrations of BMAA are required to yield neurological damage and even motor neuron death. The extent of the risk to humans from direct exposure of free BMAA in these waters remains unknown, but may be significant, and the exposure via bioaccumulation and biomagnification may be even greater.

\section{Conclusion}

The LC-MS method presented here shows an adequate lower limit of detection and quantification and can be used for the 
rapid and reproducible detection and quantification of naturally occurring BMAA, both in its free form within a complex organic matrix or in its protein associated form. The method is relatively simple and has the advantages of being standardised by the supplier and allowing the use of various analytical platforms. Additionally, the method is extremely robust with regard to multiple users and minor variations in use. Whether analysing water or biomass hydrolysate, we recommend dilution to the lower limits of quantification for accurate quantification in a matrix, to avoid matrix effects, particularly competition of SPE binding during derivatisation. Of the South African cyanobacteria tested with LC-MS, $80 \%$ contained BMAA. Again, no taxonomic or geographic variation in BMAA content or ratio of free to protein-associated BMAA was observed.

The increasing body of evidence linking BMAA to neurodegenerative diseases, and the potential for exposure to BMAA from cyanobacterial sources, suggests the need for routine monitoring, the development and adoption of safety guidelines for BMAA, and further exposure and epidemiological studies.

\section{References}

BANACK SA and COX PA (2003) Biomagnification of cycad neurotoxins in flying foxes: implications for ALS-PDC in Guam. Neurol. 61 387-389.

BANACK SA, MURCH SJ and COX PA (2006) Neurotoxic flying foxes as dietary items for the Chamorro people, Marianas Islands. J. Ethnopharmacol. 106 (1) 97-104.

BANACK SA, JOHNSON HE, CHENG R and COX PA (2007) Production of the Neurotoxin BMAA by a marine cyanobacterium. Mar. Drugs 5 180-196.

COX PA and SACKS OW (2002) Cycad neurotoxin, consumption of flying foxes, and ALS/PDC disease in Guam. Neurol. 58 956-959.

COX PA, BANACK SA and MURCH SJ (2003) Biomagnification of cyanobacterial neurotoxins and neurodegenerative disease among the Chamorro people of Guam. PNAS 100 13380-13383.

COX PA, BANACK SA, MURCH SJ, RASMUSSEN U, TIEN G, BIDIGARE RR, METCALF J, MORRISON LF, CODD GA and BERGMAN B (2005) Diverse taxa of cyanobacteria produce$\beta$ - $N$-methylamino-L-alanine, a neurotoxic amino acid. PNAS 102 5074-5078.

DUNCAN MW, STEELE JC, KOPIN IJ and MARKEY SP (1990) 2-Amino-3-(methylamino)-propionic acid (BMAA) in cycad flour: an unlikely cause of amyotrophic lateral sclerosis and parkinsonism-dementia of Guam. Neurol. 40 767-772.

ESTERHUIZEN M and DOWNING TG (2008) $\beta$ - $N$-methylamino-1alanine (BMAA) in novel South African cyanobacterial isolates. Ecotoxicol. Environ. Saf. 71 309-313.

FAASSEN EJ, GILLISSEN F, ZWEERS HAJ and LÜRLING M (2009) Detection of the neurotoxins BMAA ( $\beta$-N-methylaminoL-alanine) and DAB ( $\alpha$ - $\gamma$-diaminobutyric acid) by LC-MS/MS in Dutch urban waters with cyanobacterial blooms. Amytrophic Lateral Sclerosis 2009 (2) 79-84.

KRÜGER T, MÖNCH B, OPPENHÄUSER S and LUCKAS B (2010) LC-MS/MS determination of the isomeric neurotoxins BMAA ( $\beta$-N-methylamino-L-alanine) and DAB (2,4-diaminobutyric acid) in cyanobacteria and seeds of Cycas revoluta and Lathyrus latifolius. Toxicon 55 547-557.

KUSHNIR MM and BERGQUIST J (2009) Beta-methylamino-Lalanine analysis by liquid chromatography tandem mass spectrometry with iTRAQ as the derivative. Eur. J. Mass. Spectrom. 15 (3) 439-43.

LOBNER D, PIANA PM, SALOUS AK and PEOPLES RW (2007) $\beta$ - $N$-methylamino-L-alanine enhances neurotoxicity through multiple mechanisms. Neurobiol. Dis. 25 360-366.

METCALF JS, BANACK SA, LINDSAY J, MORRISON LF, COX PA and CODD GA (2008) Co-occurrence of $\beta$-N-methylamino-Lalanine, a neurotoxic amino acid with other cyanobacterial toxins in British waterbodies, 1990-2004. Environ. Microbiol. 10 702-708.

MONSON CS, BANACK SA and COX PA (2003) Conservation implications of Chamorro consumption of flying foxes as a possible cause of ALS-PDC in Guam. Conserv. Biol. 17 678-686.

MONTINE TJ, LI K, PERL DP and GALASKO D (2005) Lack of B-methylamino-l-alanine in brain from controls, $\mathrm{AD}$, or Chamorros with PDC. Neurol. 65 768-776.

MURCH SJ, COX PA, BANACK SA, STEELE JC and SACKS OW (2004a) Occurrence of $\beta$ - $N$-methylamino-l-alanine (BMAA) in ALS/PDC patients from Guam. Acta Neurol. Scand. 110 (4) 267-269.

MURCH SJ, COX PA and BANACK SA (2004b) A mechanism for slow release of biomagnified cyanobacterial neurotoxins and neurodegenerative disease in Guam. PNAS 101 12228-12231.

PABLO J, BANACK SA, COX PA, JOHNSON TE, PAPAPETROPOULAS S, BRADLEY W, BUCK A and MASH DC (2009) Cyanobacterial neurotoxin BMAA in ALS and Alzheimer's disease. Acta Neurol. Scand. 120 216-225.

RAO SD, BANACK SA, COX PA and WEISS JH (2006) BMAA selectively injures motor neurons via AMPA/kainate receptor activation. Exp. Neurol. 201 244-252.

ROSÉN J and HELLENÄS K-E (2008) Determination of the neurotoxin BMAA ( $\beta-N$-methylamino-L-alanine) in cycad seed and cyanobacteria by LC-MS/MS (liquid chromatography tandem mass spectrometry). Analyst 133 1785-1789.

RIPPKA R (1988) Isolation and purification of cyanobacteria. Methods Enzymol. 167 3-28.

SNYDER LR, HOGGARD JC, MONTINE JJ and SYNOVEC RE (2010) Development and application of a comprehensive twodimensional gas chromatography with time-of-flight mass spectrometry method for the analysis of L-beta-methylamino-L-alanine in human tissue. J. Chromatogr. 1217 4639-4647.

SPÁČIL Z, ERIKSSON J, JONASSON S, RASMUSSEN U, ILAG LL and BERGMAN B (2010) Analytical protocol for identification of BMAA and DAB in biological samples. Analyst 135 127-132.

VEGA A and BELL EA (1967) $\alpha$-Amino- $\beta$-methylaminopropionic acid, a new amino acid from seeds of Cycas circinalis. Phytochem. 6 (5) $759-762$ 